\title{
Extraction of the valence transversity distributions from SIDIS data
}

\author{
M. Anselmino $\odot,{ }^{1,2, *}$ R. Kishore $\odot,{ }^{3, \dagger}$ and A. Mukherjee ${ }^{3, *}$ \\ ${ }^{1}$ Dipartimento di Fisica, Università di Torino, Via Pietro Giuria 1, I-10125 Torino, Italy \\ ${ }^{2}$ INFN, Sezione di Torino, Via Pietro Giuria 1, I-10125 Torino, Italy \\ ${ }^{3}$ Department of Physics, Indian Institute of Technology Bombay, Mumbai 400076, India
}

(Received 15 September 2020; accepted 26 October 2020; published 12 November 2020)

\begin{abstract}
The transversity distribution for $u$ and $d$ quarks is usually extracted from data on spin asymmetries in semi-inclusive deep inelastic scattering (SIDIS): however, due to its chiral odd nature, it has to be coupled to another chiral odd function, typically the Collins or the dihadron fragmentation function. A recent suggestion of considering SIDIS data involving ratios of spin asymmetries and avoiding a knowledge of the Collins function is briefly discussed. New measurements, involving ratios of cross sections, are suggested.
\end{abstract} They would allow a direct extraction of the transversity ratio, $h_{1}^{d_{V}} / h_{1}^{u_{V}}$. Numerical estimates are given.

DOI: 10.1103/PhysRevD.102.096012

\section{INTRODUCTION AND FORMALISM}

The quark transversity distribution, $h_{1}^{q}(x)$ or $\Delta_{T}^{q}(x)$, is the least known of the three basic parton distributions which describe the 1-dimensional collinear representation of the partonic nucleon structure (for a recent review paper, see Ref. [1]). It is of great interest, as its integral is related to the tensor charge, a fundamental quantity which can be computed in lattice QCD [2-6]. Because it is chiral odd, information on the transversity distribution can be obtained through observables which involve another chiral odd function. This is usually done through spin asymmetries in semi-inclusive deep inelastic scattering (SIDIS) processes, which are given by a convolution of the transversity distribution and the transverse momentum dependent fragmentation function (TMD-FF) introduced by Collins [7]. Indeed the first extraction of $\Delta_{T}^{u}(x)$ and $\Delta_{T}^{d}(x)$ was obtained in this way [8-11]. Similar results were obtained by coupling the transversity distribution with a dihadron fragmentation function [12-14].

Let us briefly recall the formalism adopted to extract the transversity distributions from SIDIS data, through Collins asymmetries. Following Refs. [15,16], where all details can be found, the differential cross section for the semiinclusive production of a hadron $h$, in the current fragmentation region, from the collision of an unpolarized

\footnotetext{
*mauro.anselmino@to.infn.it

†raj.theps@gmail.com

asmita@phy.iitb.ac.in
}

Published by the American Physical Society under the terms of the Creative Commons Attribution 4.0 International license. Further distribution of this work must maintain attribution to the author(s) and the published article's title, journal citation, and DOI. Funded by SCOAP. lepton beam off a transversely polarized target can be written, in the deeply inelastic regime, as follows (see Eq. (79) of Ref. [16]):

$$
\begin{aligned}
& \frac{\mathrm{d} \sigma^{\ell p\left(S_{T}\right) \rightarrow \ell^{\prime} h X}}{\mathrm{~d} x_{B} \mathrm{~d} Q^{2} \mathrm{~d} z_{h} \mathrm{~d}^{2} \boldsymbol{P}_{T} \mathrm{~d} \phi_{S}} \\
& \quad=\frac{2 \alpha^{2}}{Q^{4}}\left\{\frac{1+(1-y)^{2}}{2} F_{U U}+\cdots\right. \\
& \left.\quad+\left[(1-y) \sin \left(\phi_{h}+\phi_{S}\right) F_{U T}^{\sin \left(\phi_{h}+\phi_{S}\right)}+\cdots\right]\right\},
\end{aligned}
$$

where we have omitted all terms which do not contribute to the Collins asymmetry. $x_{B}, y, z_{h}$ and $Q$ are the usual SIDIS variables. The quark momentum inside the target (with momentum $\boldsymbol{p}$ ) is $\boldsymbol{q}=\boldsymbol{x p}+\boldsymbol{k}_{\perp}$, while the momentum of the final hadron generated by the fragmentation of the scattered quark (with momentum $\boldsymbol{q}^{\prime}$ ) is $\boldsymbol{P}_{h}=z \boldsymbol{q}^{\prime}+\boldsymbol{p}_{\perp}$. Notice that, at order $k_{\perp} / Q, x_{B}=x$ and $z_{h}=z$. $\boldsymbol{P}_{T}$ is the hadron transverse momentum in the $\gamma^{*}$-nucleon c.m. frame and, again at order $k_{\perp} / Q$, is given by $\boldsymbol{P}_{T}=\boldsymbol{k}_{\perp}+z \boldsymbol{p}_{\perp} \cdot \phi_{h}$ and $\phi_{S}$ are, respectively, the azimuthal angle of the observed hadron and of the target polarization vector with respect to the leptonic plane. The subscript $U T$ in the structure functions $F$ reminds that we are considering the case of an unpolarized lepton beam and a transversely polarized nucleon target ( $U U$ refers to the unpolarized situation).

In the SIDIS case, the asymmetries are often expressed through their azimuthal moments,

$$
A_{U T}^{W\left(\phi_{h}, \phi_{S}\right)}=2 \frac{\int d \phi_{h} d \phi_{S}\left[d \sigma^{\uparrow}-d \sigma^{\downarrow}\right] W\left(\phi_{h}, \phi_{S}\right)}{\int d \phi_{h} d \phi_{S}\left[d \sigma^{\uparrow}+d \sigma^{\downarrow}\right]},
$$

where $W\left(\phi_{h}, \phi_{S}\right)$ is the appropriate azimuthal weight function required in order to isolate the specific 
contribution of interest and $d \sigma^{\uparrow, \downarrow}$ is the differential cross section of Eq. (1) with $S_{T}=\uparrow, \downarrow$ denoting, respectively, a transverse polarization with azimuthal angle $\phi_{S}$ and $\phi_{S}+\pi$. Then we simply have

$$
d \sigma^{\uparrow}-d \sigma^{\downarrow}=\frac{2 \alpha^{2}}{Q^{4}}\left\{2(1-y) \sin \left(\phi_{h}+\phi_{S}\right) F_{U T}^{\sin \left(\phi_{h}+\phi_{S}\right)}+\cdots\right\},
$$

$$
d \sigma^{\uparrow}+d \sigma^{\downarrow}=\frac{2 \alpha^{2}}{Q^{4}}\left\{\left[1+(1-y)^{2}\right] F_{U U}+\cdots\right\} .
$$

As the Collins effect generates a $\sin \left(\phi_{h}+\phi_{S}\right)$ modulation, we find that the azimuthal moment (2) of the Collins asymmetry in SIDIS processes is given by, from Eqs. (3) and (4):

$$
A_{U T}^{\sin \left(\phi_{h}+\phi_{S}\right)}=\frac{2(1-y) F_{U T}^{\sin \left(\phi_{h}+\phi_{S}\right)}}{\left[1+(1-y)^{2}\right] F_{U U}} \equiv D_{N N} \frac{F_{U T}^{\sin \left(\phi_{h}+\phi_{S}\right)}}{F_{U U}}
$$

where $D_{N N}=2(1-y) /\left[1+(1-y)^{2}\right]$ is the quark depolarization factor. $F_{U U}$ can be expressed as a convolution (meaning $\boldsymbol{k}_{\perp}$ and $\boldsymbol{p}_{\perp}$ integrations) of transverse momentum dependent unpolarized distribution and fragmentation functions, while $F_{U T}^{\sin \left(\phi_{h}+\phi_{S}\right)}$ is a convolution of transversity distributions and Collins fragmentation functions (precise expressions can be found, for example, in Eqs. (63) and (75) of Ref. [16]). $A_{U T}^{\sin \left(\phi_{h}+\phi_{S}\right)}$ is the quantity experimentally measured, which relates data to a combination of the unknown transversity and Collins functions.

It is useful, for a better understanding and further use in Secs. II and III, to give here explicit expressions of $F_{U U}$ and $F_{U T}^{\sin \left(\phi_{h}+\phi_{S}\right)}$, based on particular parametrizations of the various TMD involved (see Ref. [17] for a definition of all variables and a complete collection of all details):

$$
\begin{gathered}
F_{U U}=\sum_{q} e_{q}^{2} f_{q / p}(x) D_{h / q}(z) \frac{e^{-P_{T}^{2} /\left\langle P_{T}^{2}\right\rangle}}{\pi\left\langle P_{T}^{2}\right\rangle} \equiv \sum_{q} e_{q}^{2} f_{q / p}(x) D_{h / q}(z) A\left(P_{T}\right) \\
F_{U T}^{\sin \left(\phi_{h}+\phi_{S}\right)}=\sum_{q} e_{q}^{2} h_{1}^{q}(x) \Delta^{N} D_{h / q^{\uparrow}}(z) \sqrt{\frac{e}{2}} \frac{P_{T}}{M_{C}} \frac{\left\langle p_{\perp}^{2}\right\rangle_{C}^{2}}{\left\langle p_{\perp}^{2}\right\rangle} \frac{e^{-P_{T}^{2} /\left\langle P_{T}^{2}\right\rangle_{T}}}{\pi\left\langle P_{T}^{2}\right\rangle_{T}^{2}} \equiv \sum_{q} e_{q}^{2} h_{1}^{q}(x) \Delta^{N} D_{h / q^{\uparrow}}(z) B\left(P_{T}\right)
\end{gathered}
$$

where $f_{q / p}$ and $D_{h / q}$ are the usual collinear parton distribution function (PDFs) and FFs, while $\Delta^{N} D_{h / q^{\uparrow}}(z)$ is the $z$ dependent part of the Collins functions. Notice that these expressions assume, as it is usually done in the phenomenological study of the transversity distributions from SIDIS data, a factorization, in the TMDs, of the $x, z$, $k_{\perp}$ and $p_{\perp}$ dependences; the latter are taken to be Gaussian, and the $A\left(P_{T}\right)$ and $B\left(P_{T}\right)$ functions reflect their parameters. Different choices of the parameters and different forms of the factorized TMDs would not affect the general conclusions of the next sections.

\section{SUGGESTED MEASUREMENTS}

In a recent paper [18] a suggestion was made which could avoid, in the extraction of the transversity distributions $h_{1}^{q}(x)$ from SIDIS data, a dependence on the Collins functions. It simply amounts to introduce particular asymmetries involving combinations of cross sections measured for different targets and different final hadrons; in these observables the Collins functions cancel out and one remains with ratios of transversity distributions, in addition to unpolarized PDFs and FFs.

In order to describe the suggestion of Ref. [18], let us follow their notations and rewrite Eq. (1) as

$$
\sigma_{t}^{ \pm}=\sigma_{0, t}^{ \pm}+\sin \left(\phi_{h}+\phi_{S}\right) D_{N N} \sigma_{C, t}^{ \pm}+\cdots
$$

where the subscript $t$ indicates the kind of target ( $p$ for proton, $n$ for neutron and $d$ for deuteron) and the superscript + or - refers to positive or negative pions. By comparing Eqs. (1) and (8), one can easily extract the expressions of $\sigma_{0}$ and $\sigma_{C}$ :

$$
\begin{aligned}
& \sigma_{0}=\frac{\alpha^{2}}{Q^{4}}\left[1+(1-y)^{2}\right] F_{U U} \\
& \sigma_{C}=\frac{\alpha^{2}}{Q^{4}}\left[1+(1-y)^{2}\right] F_{U T}^{\sin \left(\phi_{h}+\phi_{S}\right)} .
\end{aligned}
$$

In addition, in Ref. [18], it is taken as a measure of the Collins asymmetry the ratio

$$
A_{C}=\frac{\sigma_{C}}{\sigma_{0}}=\frac{F_{U T}^{\sin \left(\phi_{h}+\phi_{S}\right)}}{F_{U U}}=\frac{1}{D_{N N}} A_{U T}^{\sin \left(\phi_{h}+\phi_{S}\right)},
$$

which only differs by the usually measured asymmetry by the $1 / D_{N N}$ factor.

Using the expressions for $F_{U U}$ and $F_{U T}^{\sin \left(\phi_{h}+\phi_{S}\right)}$ given in Eqs. (6) and (7) (considering, for the moment, the case of a generic final hadron $h$ and a proton target) one has 


$$
\begin{gathered}
\sigma_{C, p}^{h}=\frac{\alpha^{2}}{Q^{4}}\left[1+(1-y)^{2}\right] \sum_{q} e_{q}^{2} h_{1}^{q}(x) \Delta^{N} D_{h / q^{\uparrow}}(z) B\left(P_{T}\right) \\
\sigma_{0, p}^{h}=\frac{\alpha^{2}}{Q^{4}}\left[1+(1-y)^{2}\right] \sum_{q} e_{q}^{2} f_{q / p}(x) D_{h / q}(z) A\left(P_{T}\right) .
\end{gathered}
$$

Following a previous similar suggestion for helicity distributions [19] in Ref. [18] it is defined a difference asymmetry as

$$
A_{D, t} \equiv \frac{\sigma_{C, t}^{+}-\sigma_{C, t}^{-}}{\sigma_{0, t}^{+}+\sigma_{0, t}^{-}}=\frac{\sigma_{0, t}^{+}}{\sigma_{0, t}^{+}+\sigma_{0, t}^{-}} A_{C, t}^{+}-\frac{\sigma_{0, t}^{-}}{\sigma_{0, t}^{+}+\sigma_{0, t}^{-}} A_{C, t}^{-}
$$

where the second equality strictly holds only in case the Collins angle acceptance is the same for positive and negative particles. The quantity given in Eq. (13) can be obtained from available data on the Collins symmetry and the unpolarized cross section.

Assuming isospin symmetry and introducing favored and disfavored fragmentation and Collins functions:

$$
\begin{gathered}
f_{u / p}=f_{d / n} \equiv f_{1}^{u} \quad f_{d / p}=f_{u / n} \equiv f_{1}^{d} \quad f_{\bar{u} / p}=f_{\bar{d} / n} \equiv f_{1}^{\bar{u}} \quad f_{\bar{d} / p}=f_{\bar{u} / n} \equiv f_{1}^{\bar{d}} \\
f_{s / p}=f_{s / n} \equiv f_{1}^{s} \quad f_{\bar{s} / p}=f_{\bar{s} / n} \equiv f_{1}^{\bar{s}} \\
D_{\pi^{+} / u}=D_{\pi^{-} / d}=D_{\pi^{+} / \bar{d}}=D_{\pi^{-} / \bar{u}} \equiv D_{1, \text { fav }} \\
D_{\pi^{+} / \bar{u}}=D_{\pi^{-} / \bar{d}}=D_{\pi^{+} / d}=D_{\pi^{-} / u} \equiv D_{1, \text { dis }} \quad D_{\pi^{ \pm} / s, \bar{s}} \equiv D_{1, s} \\
\Delta^{N} D_{\pi^{+} / u^{\uparrow}}=\Delta^{N} D_{\pi^{-} / d^{\uparrow}}=\Delta^{N} D_{\pi^{+} / \bar{d}^{\uparrow}}=\Delta^{N} D_{\pi^{-} / \bar{u}^{\uparrow}} \equiv \Delta^{N} D_{\mathrm{fav}} \\
\Delta^{N} D_{\pi^{+} / \bar{u}^{\uparrow}}=\Delta^{N} D_{\pi^{-} / \bar{d}^{\uparrow}}=\Delta^{N} D_{\pi^{+} / d^{\uparrow}}=\Delta^{N} D_{\pi^{-} / u^{\uparrow}} \equiv \Delta^{N} D_{\mathrm{dis}} \quad \Delta^{N} D_{\pi^{ \pm} / s, \bar{s}}=\Delta^{N} D_{1, s},
\end{gathered}
$$

one can work out, from Eqs. (11) and (12), the expressions of $\sigma_{C, t}^{ \pm}$and $\sigma_{0, t}^{ \pm}$, as done in Ref. [18]. Dropping the common factor $(1 / 9)\left(\alpha^{2} / Q^{4}\right)\left[1+(1-y)^{2}\right] B\left(P_{T}\right)$, one has

$$
\begin{gathered}
\sigma_{C, p}^{+} \sim\left[\left(4 h_{1}^{u}+h_{1}^{\bar{d}}\right) \Delta^{N} D_{\mathrm{fav}}+\left(4 h_{1}^{\bar{u}}+h_{1}^{d}\right) \Delta^{N} D_{\mathrm{dis}}+\left(h_{1}^{s}+h_{1}^{\bar{s}}\right) \Delta^{N} D_{1, s}\right] \\
\sigma_{C, p}^{-} \sim\left[\left(4 h_{1}^{u}+h_{1}^{\bar{d}}\right) \Delta^{N} D_{\mathrm{dis}}+\left(4 h_{1}^{\bar{u}}+h_{1}^{d}\right) \Delta^{N} D_{\mathrm{fav}}+\left(h_{1}^{s}+h_{1}^{\bar{s}}\right) \Delta^{N} D_{1, s}\right] \\
\sigma_{C, d}^{+} \sim\left[\left(h_{1}^{u}+h_{1}^{d}\right)\left(4 \Delta^{N} D_{\mathrm{fav}}+\Delta^{N} D_{\mathrm{dis}}\right)+\left(h_{1}^{\bar{u}}+h_{1}^{\bar{d}}\right)\left(\Delta^{N} D_{\mathrm{fav}}+4 \Delta^{N} D_{\mathrm{dis}}\right)+2\left(h_{1}^{s}+h_{1}^{\bar{s}}\right) \Delta^{N} D_{1, s}\right] \\
\sigma_{C, d}^{-} \sim\left[\left(h_{1}^{u}+h_{1}^{d}\right)\left(4 \Delta^{N} D_{\mathrm{dis}}+\Delta^{N} D_{\mathrm{fav}}\right)+\left(h_{1}^{\bar{u}}+h_{1}^{\bar{d}}\right)\left(\Delta^{N} D_{\mathrm{dis}}+4 \Delta^{N} D_{\mathrm{fav}}\right)+2\left(h_{1}^{s}+h_{1}^{\bar{s}}\right) \Delta^{N} D_{1, s}\right]
\end{gathered}
$$

and, dropping the common factor $(1 / 9)\left(\alpha^{2} / Q^{4}\right)\left[1+(1-y)^{2}\right] A\left(P_{T}\right)$,

$$
\begin{gathered}
\sigma_{0, p}^{+} \sim\left[\left(4 f_{1}^{u}+f_{1}^{\bar{d}}\right) D_{1, \mathrm{fav}}+\left(4 f_{1}^{\bar{u}}+f_{1}^{d}\right) D_{1, \mathrm{dis}}+\left(f_{1}^{s}+f_{1}^{\bar{s}}\right) D_{1, s}\right] \\
\sigma_{0, p}^{-} \sim\left[\left(4 f_{1}^{u}+f_{1}^{\bar{d}}\right) D_{1, \mathrm{dis}}+\left(4 f_{1}^{\bar{u}}+f_{1}^{d}\right) D_{1, \mathrm{fav}}+\left(f_{1}^{s}+f_{1}^{\bar{s}}\right) D_{1, s}\right] \\
\sigma_{0, d}^{+} \sim\left[\left(f_{1}^{u}+f_{1}^{d}\right)\left(4 D_{1, \mathrm{fav}}+D_{1, \mathrm{dis}}\right)+\left(f_{1}^{\bar{u}}+f_{1}^{\bar{d}}\right)\left(D_{1, \mathrm{fav}}+4 D_{1, \mathrm{dis}}\right)+2\left(f_{1}^{s}+f_{1}^{\bar{s}}\right) D_{1, s}\right] \\
\sigma_{0, d}^{-} \sim\left[\left(f_{1}^{u}+f_{1}^{d}\right)\left(4 D_{1, \mathrm{dis}}+D_{1, \mathrm{fav}}\right)+\left(f_{1}^{\bar{u}}+f_{1}^{\bar{d}}\right)\left(D_{1, \mathrm{dis}}+4 D_{1, \mathrm{fav}}\right)+2\left(f_{1}^{s}+f_{1}^{\bar{s}}\right) D_{1, s}\right],
\end{gathered}
$$

where we have used $\sigma_{d}=\sigma_{p}+\sigma_{n}$.

The suggestion of Ref. [18] is that of measuring ratios of the difference asymmetries defined in Eq. (13). This is because, as it can be see from the above expressions of $\sigma_{C}$ and $\sigma_{0}$, the differences $\left(\sigma_{C, p}^{+}-\sigma_{C, p}^{-}\right)$and $\left(\sigma_{C, d}^{+}-\sigma_{C, d}^{-}\right)$have the same dependence ( $\Delta^{N} D_{\text {fav }}-\Delta^{N} D_{\text {dis }}$ ) on the Collins function, which then cancels out in the ratio. Notice that in the differences $\left(\sigma_{C, t}^{+}-\sigma_{C, t}^{-}\right)$the contributions from strange quarks drop out. Then one has 


$$
R_{D, d / p} \equiv \frac{A_{D, d}}{A_{D, p}}=3\left[\frac{\left(4 f_{1}^{u}+4 f_{1}^{\bar{u}}+f_{1}^{d}+f_{1}^{\bar{d}}\right)\left(D_{1, \mathrm{fav}}+D_{1, \mathrm{dis}}\right)+2\left(f_{1}^{s}+f_{1}^{\bar{s}}\right) D_{1, s}}{5\left(f_{1}^{u}+f_{1}^{\bar{u}}+f_{1}^{d}+f_{1}^{\bar{d}}\right)\left(D_{1, \mathrm{fav}}+D_{1, \mathrm{dis}}\right)+4\left(f_{1}^{s}+f_{1}^{\bar{s}}\right) D_{1, s}}\right] \frac{h_{1}^{u_{v}}+h_{1}^{d_{v}}}{4 h_{1}^{u_{v}}-h_{1}^{d_{v}}}
$$

as in Eq. (16) of Ref. [18] and where $h_{1}^{q_{v}}=h_{1}^{q}-h_{1}^{\bar{q}}$.

Notice that Eq. (28) further simplifies if one neglects the contribution of $s$ quarks. It gives a direct access, assuming one knows the unpolarized PDFs and FFs, to the ratio of the $u$ and $d$ transversity distributions.

The advantage of suggesting a measurement of $R_{D, d / p}$, apart from its direct relation to $h_{1}^{d_{v}} / h_{1}^{u_{v}}$, is that it can be obtained from available data on the Collins asymmetry and the unpolarized cross section $\sigma_{0}$. However, it has the disadvantage that it is a ratio of two very small quantities, $A_{D, d}$ and $A_{D, p}$, both with large relative errors. Then, their ratio is bound to have huge uncertainties, as pointed out also in Refs. [18] and [20]. The situation might improve with the planned next COMPASS run with a deuteron target [20]. Such a run might offer new possibilities, like the measurement of cross sections. In the next section we suggest another way of obtaining direct information on the ratio of transversity distribution for $u$ and $d$ valence quarks.

\section{NEW MEASUREMENTS, NUMERICAL ESTIMATES AND CONCLUSIONS}

If one could measure the $\sin \left(\phi_{h}+\phi_{S}\right)$ modulation of the SIDIS cross section (1), that is $\sigma_{C}$, for different targets and positive and negative pions, then one could build the ratio

$$
R_{C, d / p} \equiv \frac{\sigma_{C, d}^{+}-\sigma_{C, d}^{-}}{\sigma_{C, p}^{+}-\sigma_{C, p}^{-}}
$$

which, from Eqs. (20)-(23), has a very simple partonic interpretation:

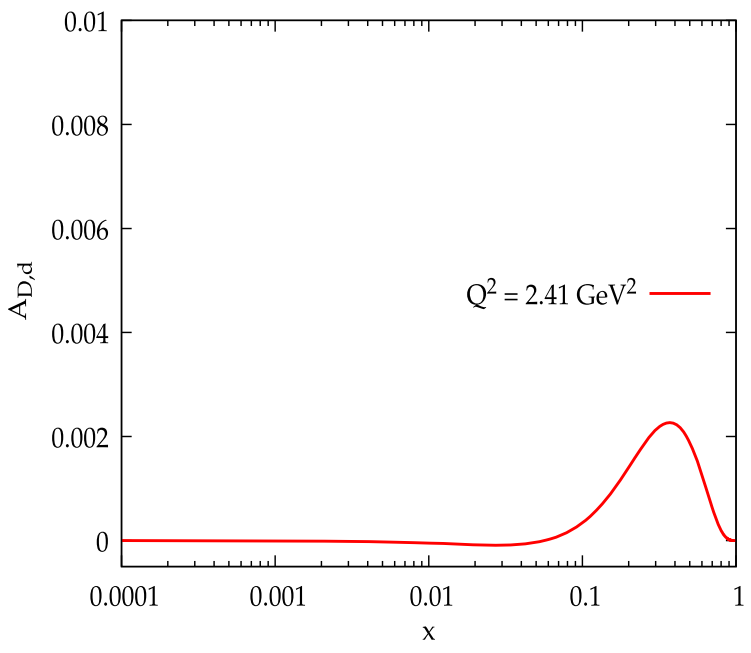

$$
R_{C, d / p}=3 \frac{h_{1}^{u_{v}}+h_{1}^{d_{v}}}{4 h_{1}^{u_{v}}-h_{1}^{d_{v}}}=3 \frac{1+\frac{h_{1}^{d_{v}}}{h_{1}^{u_{v}}}}{4-\frac{h_{1}^{d_{v}}}{h_{1}^{u_{v}}}}
$$

Not only the dependence on the Collins functions cancel out, like in Eq. (28), but also the dependence on the unpolarized PDFs and FFs. Moreover, $R_{C, d / p}$, compared to $R_{D, d / p}$, is not a ratio of two small quantities, with a consequent smaller uncertainty. This is essentially due to the fact that the $A_{D}$ 's are related to a ratio $\sigma_{C} / \sigma_{0} \sim\left(h_{1} \Delta^{N} D\right) /\left(f_{1} D\right)$, while the numerator and denominator of $R_{C}$ are simply proportional to $\sigma_{C} \sim\left(h_{1} \Delta^{N} D\right)$.

A similarly simple expression holds if one measures the SIDIS cross section off a neutron target, possibly at JLab [21]:

$$
R_{C, n / p}=\frac{4 h_{1}^{d_{v}}-h_{1}^{u_{v}}}{4 h_{1}^{u_{v}}-h_{1}^{d_{v}}}=\frac{4 \frac{h_{1}^{d_{v}}}{h_{1}^{u_{v}}}-1}{4-\frac{h_{1}^{d_{v}}}{h_{1}^{u_{v}}}} .
$$

We can give some estimates for the suggested asymmetries, based on our actual knowledge of the transversity distributions. We use the simple parametrization for the transversity distributions and the Collins functions as in Eqs. (9)-(12) of Ref. [10]. In that reference, the best fit free parameters for the $u$ and $d$ quarks transversity distributions functions and for the favored and disfavored Collins fragmentation functions have been extracted by fitting HERMES, COMPASS and Belle data. For our plots we use the values given in Table II of Ref. [10]. Notice that the

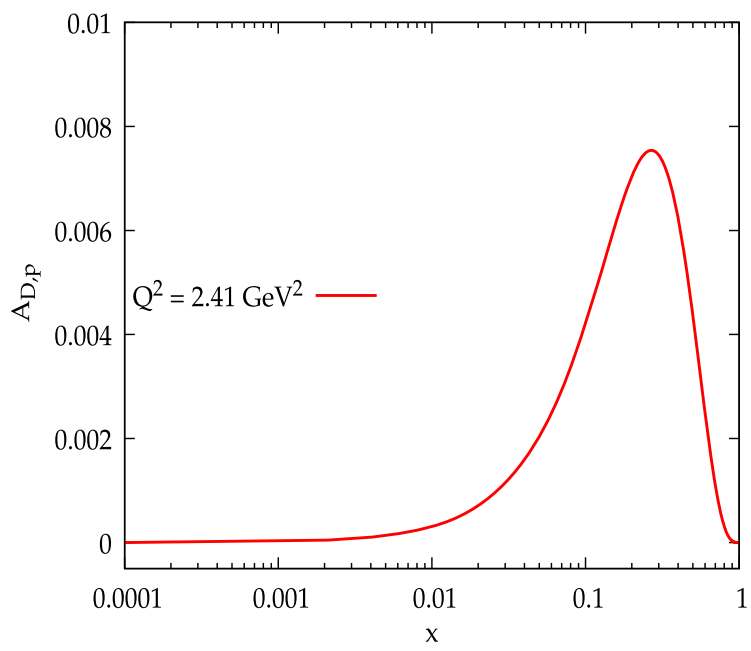

FIG. 1. Plot of the difference asymmetries $A_{D, d}$ (left panel) and $A_{D, p}$ (right panel) vs $x$ at $Q^{2}=2.41 \mathrm{GeV}^{2}$. The $z$ and $P_{T}$ variables are integrated in the ranges $0.1<z<1$ and $0<P_{T}<5 \mathrm{GeV}$. 

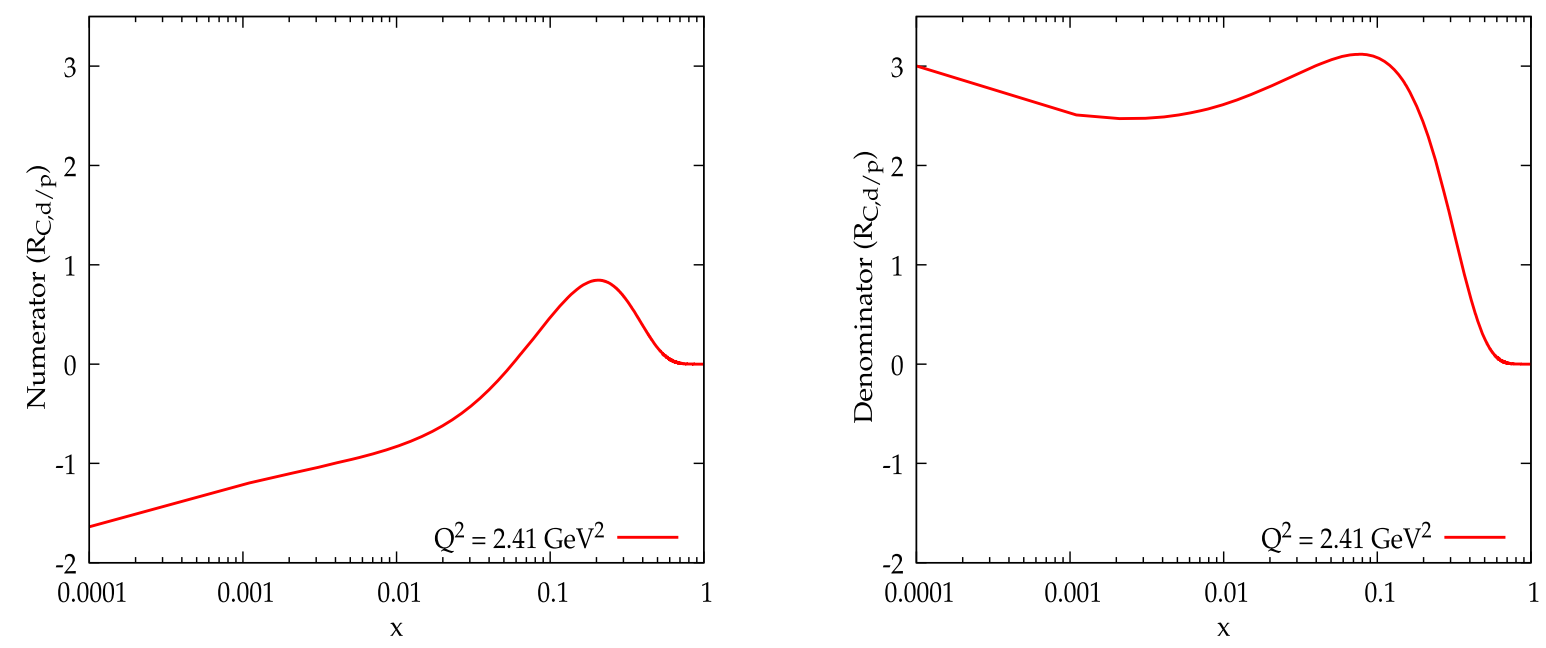

FIG. 2. Plot of the numerator (left panel) and denominator (right panel) of $R_{C, d / p}$ vs $x$ at $Q^{2}=2.41$. The $z$ variable is integrated in the ranges $0.1<z<1$.

transversity distributions for $\bar{u}$ and $\bar{d}$ are assumed to be negligible.

In Fig. 1 we plot $A_{D, d}(x)$ and $A_{D, p}(x)$ defined in Eq. (13), using Eqs. (20)-(27) where we have reinserted all factors. The $y$ dependence cancels out, while the $P_{T}$ and $z$ variables are integrated in the ranges $0<P_{T}<5 \mathrm{GeV}$ and $0.1<$ $z<1$. We have fixed $Q^{2}=2.41 \mathrm{GeV}^{2}$, which is the $Q^{2}$ value of the results of Ref. [10]. The PDFs are taken from MSTW2008 [22] and the unpolarized pion FFs from Ref. [23]; for the helicity distributions we refer to Ref. [24]. Very similar results could be obtained simply using directly in Eq. (13) the expressions (20)-(27), and integrating over $z$.

In Fig. 2 we show the numerator and denominator of $R_{C, d / p}$, respectively $\left(\sigma_{C, d}^{+}-\sigma_{C, d}^{-}\right)$and $\left(\sigma_{C, p}^{+}-\sigma_{C, p}^{-}\right)$as obtained from Eqs. (20)-(23). The $z$ variable is integrated between 0.1 and 1. A similar plot could be shown for the numerator of $R_{C, n / p}$.

These estimates clearly confirm our expectations. The difference asymmetries, $A_{D, d}(x)$ and $A_{D, p}(x)$, available from existing data, are, however, very small; their uncertainties, due to experimental errors and difficulties, can be as large as their values; their ratio, which could avoid a knowledge of the Collins function, is bound to have huge uncertainties $[18,20]$.

Our suggested measurements of $R_{C, d / p}$ and $R_{C, n / p}$ require a knowledge of the SIDIS cross section (1) and in particular of its $\sin \left(\phi_{h}+\phi_{S}\right)$ modulation, which might be difficult. However, their TMD interpretation is much cleaner and allows a direct measurement of the ratio $h_{1}^{d_{v}} / h_{1}^{u_{v}}$, through the ratio of two quantities which can be orders of magnitude larger than the difference asymmetries.

We are confident that the simplicity of $R_{C, d / p}$ and $R_{C, n / p}$ in terms of the transversity distributions, Eqs. (30) and (31), will prompt and encourage their measurements; this could be done during the next deuteron COMPASS run, or during the ongoing JLab 12 experiments or at the future EIC facility.

\section{ACKNOWLEDGMENTS}

M. A. would like to thank the Physics Department of the Indian Institute of Technology in Mumbai (IIT Bombay), where this paper was initiated, for hospitality and support. We are grateful to F. Bradamante and V. Barone for useful comments and discussions and to U. D'Alesio for some help with the pion FFs.
[1] M. Anselmino, A. Mukherjee, and A. Vossen, Transverse spin effects in hard semi-inclusive collisions, Prog. Part. Nucl. Phys. 114, 103806 (2020).

[2] T. Bhattacharya, V. Cirigliano, S. D. Cohen, R. Gupta, H. W. Lin, and B. Yoon, Axial, scalar and tensor charges of the nucleon from $2+1+1$-flavor lattice QCD, Phys. Rev. D 94, 054508 (2016).
[3] C. Alexandrou, M. Constantinou, P. Dimopoulos, R. Frezzotti, K. Hadjiyiannakou, K.Jansen, C. Kallidonis, B. Kostrzewa, G. Koutsou, M. Mangin-Brinet, A. Vaquero Aviles-Casco, and U. Wenger, Nucleon scalar and tensor charges using lattice QCD simulations at the physical value of the pion mass, Phys. Rev. D 95, 114514 (2017); Erratum, Phys. Rev. D 96, 099906 (2017). 
[4] R. Gupta, B. Yoon, T. Bhattacharya, V. Cirigliano, Y. C. Jang, and H. W. Lin, Flavor diagonal tensor charges of the nucleon from $(2+1+1)$-flavor lattice QCD, Phys. Rev. D 98, 091501(R) (2018).

[5] N. Yamanaka, S. Hashimoto, T. Kaneko, and H. Ohki (JLQCD Collaboration), Nucleon charges with dynamical overlap fermions, Phys. Rev. D 98, 054516 (2018).

[6] M. Constantinou, A. Courtoy, M. A. Ebert, M. Engelhardt, T. Giani, T. Hobbs, T. J. Hou, A. Kusina, K. Kutak, and J. Liang et al., Parton distributions and lattice QCD calculations: toward 3D structure, arXiv:2006.08636.

[7] J.C. Collins, Fragmentation of transversely polarized quarks probed in transverse momentum distributions, Nucl. Phys. B396, 161 (1993).

[8] M. Anselmino, M. Boglione, U. D’Alesio, A. Kotzinian, F. Murgia, A. Prokudin, and C. Turk, Transversity and Collins functions from SIDIS and $e^{+} e^{-}$data, Phys. Rev. D 75, 054032 (2007).

[9] M. Anselmino, M. Boglione, U. D’Alesio, A. Kotzinian, F. Murgia, A. Prokudin, and S. Melis, Update on transversity and Collins functions from SIDIS and $e^{+} e^{-}$data, Nucl. Phys. B, Proc. Suppl. 191, 98 (2009).

[10] M. Anselmino, M. Boglione, U. D’Alesio, S. Melis, F. Murgia, and A. Prokudin, Simultaneous extraction of transversity and Collins functions from new SIDIS and $e^{+} e^{-}$ data, Phys. Rev. D 87, 094019 (2013).

[11] Z. B. Kang, A. Prokudin, P. Sun, and F. Yuan, Extraction of quark transversity distribution and Collins fragmentation functions with QCD evolution, Phys. Rev. D 93, 014009 (2016).

[12] A. Bacchetta, A. Courtoy, and M. Radici, First Glances at the Transversity Parton Distribution Through Dihadron Fragmentation Functions, Phys. Rev. Lett. 107, 012001 (2011).

[13] A. Bacchetta, A. Courtoy, and M. Radici, First extraction of valence transversities in a collinear framework, J. High Energy Phys. 03 (2013) 119.
[14] M. Radici, A. Courtoy, A. Bacchetta, and M. Guagnelli, Improved extraction of valence transversity distributions from inclusive dihadron production, J. High Energy Phys. 05 (2015) 123.

[15] A. Bacchetta, M. Diehl, K. Goeke, A. Metz, P. J. Mulders, and M. Schlegel, Semi-inclusive deep inelastic scattering at small transverse momentum, J. High Energy Phys. 02 (2007) 093.

[16] M. Anselmino, M. Boglione, U. D'Alesio, S. Melis, F. Murgia, E. R. Nocera, and A. Prokudin, General helicity formalism for polarized semi-inclusive deep inelastic scattering, Phys. Rev. D 83, 114019 (2011).

[17] M. Anselmino, M. Boglione, U. D'Alesio, F. Murgia, and A. Prokudin, Role of transverse momentum dependence of unpolarized parton distribution and fragmentation functions in the analysis of azimuthal spin asymmetries, Phys. Rev. D 98, 094023 (2018).

[18] V. Barone, F. Bradamante, A. Bressan, A. Kerbizi, A. Martin, A. Moretti, J. Matousek, and G. Sbrizzai, Transversity distributions from difference asymmetries in semiinclusive DIS, Phys. Rev. D 99, 114004 (2019).

[19] E. Christova and E. Leader, A strategy for the analysis of semiinclusive deep inelastic scattering, Nucl. Phys. B607, 369 (2001).

[20] COMPASS Collaboration, COMPASS status report 2020, CERN Report No. CERN-SPSC-2020-015/SPSC-SR-271, 2020.

[21] H. Gao, T. Liu, and Z. Zhao, The TMD program at JLab, Proc. Sci., DIS2018 (2018) 232.

[22] A. D. Martin, W. J. Stirling, R. S. Thorne, and G. Watt, Parton distributions for the LHC, Eur. Phys. J. C 63, 189 (2009).

[23] D. de Florian, R. Sassot, M. Epele, R. J. Hernandez-Pinto, and M. Stratmann, Parton-to-pion fragmentation reloaded, Phys. Rev. D 91, 014035 (2015).

[24] M. Gluck, E. Reya, M. Stratmann, and W. Vogelsang, Models for the polarized parton distributions of the nucleon, Phys. Rev. D 63, 094005 (2001). 